\title{
REFLEXIONES RESPECTO DE UN PLAN DE ESTUDIO PARA PEDAGOGÍA EN EDUCACIÓN FÍSICA CON FUNDAMENTACIÓN PARADIGMATICA EN LA CIENCIA DE LA MOTRICIDAD HUMANA
}

\section{REFLECTIONS IN RESPECT OF A CURRICULUM FOR PHYSICAL EDUCATION TEACHING GROUNDED IN HUMAN MOVEMENT SCIENCE PARADIGM}

\begin{abstract}
Portes Júnior, Moacyr; Almonacid Fierro, Aquiles Alejandro \& Castro Jaque, Cesar Augusto.
\end{abstract}
Universidad Autónoma de Chile, sede Talca, Chile.

PORTES J.M.; ALMONACID F.A.A. \& CASTRO J.C.A. (2014). Reflexiones respecto de un plan de estudio para pedagogía en educación física con fundamentación paradigmatica en la ciencia de la motricidad humana. Mot. Hum. 15(2): 124-133.

\section{RESUMEN}

En momentos de ajustes o cambios, los paradigmas que sustentan áreas sociales pueden ser afectados directamente por ellos. La Educación Física enfrenta la necesidad de establecer las acciones profesionales en el ámbito de la Educación Física escolar, considerando que o ser humano enfrenta crisis en el proceso de humanización. Para ello, la Ciencia de la Motricidad Humana viene a resignificar la acción educativo-motriz desde una mirada antropológica con énfasis en la persona humana. Modelos pedagógicos de formación del profesorado en Educación Física siguen esta tendencia. Pero enfrentan barreras establecidas por la realidad sociocultural hipocinética, de la alimentación hipercalórica, desinterés por la actividad física y por políticas públicas de salud que priorizan la acción motriz descontextualizada del proceso de humanización. Este texto presenta la realidad del establecimiento de una carrera con bases en el paradigma de la Ciencia de la Motricidad Humana, partiendo de reflexiones históricas de la Educación Física, considerando el currículo chileno para el área y termina indagando en qué rumbos ha de seguir la formación de los futuros profesionales del área de Educación Física.

Palabras Claves: Formación Profesional, Educación Física, Ciencia de la Motricidad Humana, Curriculum, Sociedad.

\section{ABSTRACT}

At adjustments moments or changes, the paradigms that they sustain social areas can be affected directly by them. The Physical Education faces the necessity to establish the professional actions in the scope of the scholar Physical Education, considering that human being faces crisis in the humanization process. For it, the Human Motricity Science comes to resignificar the educative-motor action from an anthropological glance with emphasis in the human person. Pedagogical models of formation of the teaching staff in Physical Education follow this tendency. But they face barriers established by the hypo kinetic social cultural reality, of the hyper caloric feeding, disinterestedness by the physical activity and public policies of health that prioritize the off out context motor action of the humanization process. This paper presents the reality of establishing a career with bases in the paradigm of Human Motricity Science, based on historical reflections of Physical Education, considering the Chilean curriculum area and ends in inquiring what courses must follow the training of future professionals in the area of Physical Education.

Key Words: Professional Formation, Physical Education, Human Motricity Science, Curriculum, Society. 
PORTES J.M.; ALMONACID F.A.A. \& CASTRO J.C.A. (2014). Reflexiones respecto de un plan de estudio para pedagogía en educación física con fundamentación paradigmatica en la ciencia de la motricidad humana. Mot. Hum. 15(2): 123-133.

\section{INTRODUCCIÓN}

Para comprender las razones de la existencia de asignaturas o áreas de formación dentro de los planes de estudio en el subsector de Educación Física, es menester comprender algunos principios epistemológicos del área (Moreira y Carbinatto, 2006), y seguidamente, definir los supuestos epistemológicos de la disciplina Educación Física, cuestión que tiende a ser una tarea difícil y compleja, toda vez que son diversas las perspectivas paradigmáticas que dan respuesta a la noción de ciencia que subyace en el área y a los aspectos metodológicos-didácticos que la soportan. (Bracht, 1999; Guedes, 2000; Sacardo y Hayashi, 2010; Galak y Ganbarotta, 2011; Savarí, 2012).

La Educación Física abarca una multidisciplinaridad de comprensiones y patrones epistemológicos; ratificar una epistemología única es tarea que debe pasar por varias fases de análisis del área, como también por una reflexión profunda acerca de perspectiva de hombre y del movimiento, objeto de estudio del área. (Oliveira y Porpino, 2010; Rigo, et al., 2011). Lo anterior, hasta llegar a la comprensión del ser humano que se moviliza de manera consciente hacia acciones con sentido y en busca de la trascendencia desde la corporeidad, objeto de estudio de la Motricidad Humana.

La comprensión integral del ser humano, en que basa la estructura curricular la Educación Física, está centrada en los referentes internacionales orientados por la FIEP (Federación Internacional de la Educación Física) y los componentes curriculares chilenos para la Educación Física escolar (Chile, 2013), como también, el instrumento de comprensión utilizado en las asignaturas de formación que pertenecen al plan de estudios de las carrera de Pedagogía en Educación Física en Chile, cuestión que no significa que se haga parte del modelo pedagógico de los planes de estudio de las referidas unidades.

De esta manera, la Educación Física, en los cursos de formación de profesores, debe estar contextualizada para la escuela, buscando a través de las diversas contribuciones de las ciencias biológicas, humanas, sociales, psicológicas, pedagógicas y del área deportiva, la comprensión de la Motricidad Humana como un lenguaje, comprendido y asumido, que hace entender al ser humano bajo las perspectivas de cada una de ellas.
(Costa, et al., 1996; Veloso, 2010). De la misma manera, el currículo es implementado teniendo en consideración aspectos específicos de cada una de las ciencias y áreas, que en las asignaturas del plan de estudios de los profesores en formación, reflejan esa fragmentación que se visualiza en los docentes de Educación Física; fragmentación que debiera estar siempre direccionada a la comprensión del todo, en el bien entendido que el todo es siempre a la sumatoria de las partes. (Sergio, 1996; Carrizo y Rodríguez, 2012; Braid, et al., 2012).

Esta fragmentación puede estar relacionada a los diversos paradigmas que fundamentaron las acciones en el área de Educación Física y a su vez, el establecimiento de planes de estudio para la formación de profesionales de Educación Física. De igual forma, en los Planes de Estudio, se pueden encontrar asignaturas que se basan en estos paradigmas, siendo la sumatoria de estos conocimientos que hacen de la Educación Física, lo que se tiene hoy. Estos paradigmas son el resultado del desarrollo a través de la historia, de la investigación, de la cultura social cambiante y del desarrollo específico del área de Educación Física, y que pueden ser encontrados en las áreas de: la salud (Chile, 2002; Farias, 2008; Ardoy, et al., 2010; Escalante, et al., 2011; Cuadra-Martínez, et al., 2012; Cordova, et al., 2012; Dir. Salud Int. Niñez y Ado., 2012; González, et al., 2012), lo lúdico (Marcelino, 1999; Silva, 2012), el deporte (Ubirajara, 1999; Souza Neto, et al., 2004; Rogelin, et al., 2010; Machado y Vargas, 2011), la pedagogía (Ubirajara, 1999; Lima, 2000; Boscatto y Kunz 2009; Marcon, et al., 2012), los juegos (Bougére, 1998; Figueredo González, et al., 2003; Betti, et al., 2011; Usabiaga Arruabarrena, et. al., 2012; Rivero, 2012) y por supuesto la emergente Ciencia de Motricidad Humana (Sergio, 1996; Lagos Hernández, 2011).

Esta reflexión se adscribe a la perspectiva paradigmática de la Ciencia de la Motricidad Humana (CMH), toda vez que se entiende como una ciencia que viene a resignificar la acción educativomotriz desde una mirada antropológica con énfasis en la persona humana, que considera al joven en todas sus complejas y ricas dimensiones, comprendiendo el movimiento como movimiento intencionado y expresado a través de la corporeidad. (Sergio, 1996). 
PORTES J.M.; ALMONACID F.A.A. \& CASTRO J.C.A. (2014). Reflexiones respecto de un plan de estudio para pedagogía en educación física con fundamentación paradigmatica en la ciencia de la motricidad humana. Mot. Hum. 15(2): 124-133.

\section{¿Un nuevo paradigma?}

La red internacional de investigadores en Motricidad Humana, señala que la $\mathrm{CMH}$ nace, por tanto, de tres cortes epistemológicos. El primero es el de la disciplina, la Educación Física; el segundo es el del concepto clásico de ciencia; el tercero se refiere al concepto de conocimiento. En consecuencia, la Motricidad Humana viene a representar una nueva ciencia del hombre, en la búsqueda permanente del desarrollo humano.

Desde la CMH la Educación Física es vista como un espacio generoso en la construcción de sentidos y significados, para que hombres y mujeres puedan levantar proyectos de vida a partir de la relación, y en consecuencia, construir y compartir mundos posibles teniendo como telón de fondo la humanización de las actividades deportivas y recreativas que despliega el ser humano. (Almonacid, 2012)

La comprensión que pretende la $\mathrm{CMH}$ en la Educación Física, involucra una visión más integral del ser humano. De esta manera, la Educación Física en su globalidad y la Educación Física escolar, deben ser pensadas y establecidas, de tal manera que sean capaz de responder a las necesidades individuales y sociales, relacionando las acciones pedagógicas y la práctica profesional, la formación de futuros docentes y profesionales de otras áreas de intervención, relacionadas a la Educación Física, entregando la formación y capacitación necesarias supliendo las necesidades de las competencias exigidas por la realidad social, académica y epistemológica del área. (Neira, 2012)

Los análisis hechos por Manuel Sergio (Sergio, 2003), de los caminos de la Educación Física en el contexto histórico social, indicaron un proceso que ha pasado por varias transformaciones, a raíz de la comprensión del hombre en su proceso histórico. Desde la perspectiva instrumental del cuerpo (sobrevivencia), de la representación dicotómica del mismo, del carácter racionalista del cuerpo, hasta llegar a la complejidad de la visión integradora y de sentido, indicando la evolución del pensamiento que el hombre está tejido conjuntamente, en todas sus partes que forman el todo, y de que el movimiento humano no es solo el movimiento "de per si", sino que la praxis reconocida de este ser humano, lo lleva a la acción consciente, sistémica y libre, hacia la humanización. (Freire, et al., 2011)

Esta mirada sistémica hace referencia a la unidad, a un todo integrado que es el resultado de todas las relaciones y conexiones en el trascurso del desarrollo. Lo que hace del ser humano un ser vulnerable a las influencias e interacciones que actúan en su potencial biológico, hacia la condición sociocultural que alcanza; las experiencias vivenciadas, las oportunidades entregadas, transforman, desde una perspectiva compleja la multidimensionalidad del hombre.

Otros autores, Piaget (Piaget, 1969), Vygotsky (Vygotsky, 1978), Erickson (Erickson, 1983), Bruner (Bruner, 1987), Ausubel (Ausubel, et al., 1983), Rogers (Rogers, 1961) y Maslow (Maslow, 1943), hasta la comprensión de Kolyniak (Kolyniak, 2008), relacionan más directamente la $\mathrm{CMH}$, indican la relación del hombre con el mundo, de manera intencional y llena de significados, entregan la percepción del mundo, la memoria, la proyección, la afectividad, la emoción, el raciocinio, evidenciadas por las diferentes formas de comunicación y expresión - verbal y no verbal que referido con la $\mathrm{CMH}$ es la acción intencional la que reúne las diferentes dimensiones del ser humano.

Desde la $\mathrm{CMH}$, la Educación Física es percibida, como un espacio óptimo para favorecer la construcción de los sentidos y significados que el hombre necesita para cimentar las posibilidades para su humanización. Eso por cuenta de los objetivos que están en los fundamentos del área y que a partir de un proceso educacional, permiten la apropiación de los contenidos que hacen parte de la cultura corpórea y motricia de los estudiantes, de manera que ellos tengan la posibilidad de vivenciar y analizar la motricidad en los diferentes contextos (biológicos, neuro-comportamentales, afectivosociales y socioculturales) en condiciones de alcanzar su pleno desarrollo - humanización. (Pérez Gallardo, 2004) 
PORTES J.M.; ALMONACID F.A.A. \& CASTRO J.C.A. (2014). Reflexiones respecto de un plan de estudio para pedagogía en educación física con fundamentación paradigmatica en la ciencia de la motricidad humana. Mot. Hum. 15(2): 124-133.

\section{Educación Física y Curriculum}

El establecimiento de un Plan de Estudios y de una malla curricular, debe ser hecho para orientar y dar cumplimiento de lo expresado en las bases epistemológicas del área, que es la referencia del modelo pedagógico de las carreras y que se conecta de manera directa e indirecta en los contenidos de las asignaturas que la componen, representando, así, la coherencia con el paradigma y al modelo de formación y con los referentes curriculares nacionales e internacionales. En la $\mathrm{CMH}$, las teorías que fundamentan están declaradas en las discusiones realizadas por Trigo y Montoya (Trigo y Montoya, 2006; Trigo y Montoya, 2009), en la construcción de su marco referencial.

La relación de las asignaturas del plan de estudios de carreras de Pedagogía en Educación Física, con los fundamentos de la $\mathrm{CMH}$, está definida en tres áreas. En el Área de Formación Básica, considerando que esta área está relacionada a la formación del futuro docente, de modo de trascender en sus capacidades personales y pueda comprender tanto la sociedad como al individuo que forma la sociedad, abarca la casi la totalidad de las teorías que fundamentan la $\mathrm{CMH}$, teniendo presente que el conjunto de acciones en ella, puedan ser direccionadas a la formación integral del docente en formación. La puesta en práctica de la formación profesional que busca en los estudiantes un desarrollo integral, como principio preconizado en los modelos pedagógicos de las instituciones formadoras chilenas, expresado en los planes y programas entregados por el Ministerio de Educación.

En el Área de Formación Profesional se relaciona la formación de especialidad de la carrera, que involucra asignaturas del ámbito de la biología, pedagogía y técnico-deportivo. En lo biológico, está la comprensión del ser humano en su desarrollo biofisiológico y las posibilidades de desarrollo que puede proporcionar al ser, a partir de la praxis y las inter-relaciones que estas praxis pueden aportar. Reconocer al ser humano como un ser que se hace en movimiento intencionadamente en busca de su auto superación.

En lo técnico-deportivo, elemento clave para la comprensión de los medios (contenidos prácticos) de la Educación Física escolar, y la utilización de estos medios para contribuir al desarrollo y a la transcendencia del estudiante, partiendo desde las acciones individuales, colectivas, cooperativas, competitivas, de colaboración, de oposición, y todas las manifestaciones de la cultura corporal y motora, que contribuyen de manera directa a las tres dimensiones en que los objetivos de las actividades escolares deben estar basados, conceptuales, procedimentales y actitudinales.

En el área pedagógica, están las asignaturas que han de entregar conocimientos y capacitación para que el futuro docente sea capaz de identificar, planear, programar, organizar, dirigir, coordinar, supervisar, desarrollar, evaluar y contribuir al logro de los contenidos del componente curricular/asignatura Educación Física. Favorecer las condiciones para el proceso de crecimiento y de desarrollo del ser humano y su relación con la enseñanza, en una perspectiva crítico-histórica, para ejecutar lo propuesto de las actividades físicas y los deportes dónde están presentes - los orígenes de transformación y la emancipación.

Se integran las asignaturas relacionadas a las aproximaciones e inserciones a los procesos educativos, que son las instancias de acercamiento a la realidad laboral, hasta llegar a la práctica profesional, que tiene como objetivo, desarrollar en el futuro profesional, capacidades de dominio permanente en las diferentes dimensiones epistemológicas y profesionales de su formación académica; además, tener competencias técnicas y las habilidades necesarias, para la elaboración, ejecución y evaluación de programas de actividades físicas adecuadas a los distintos momentos de la enseñanza.

Se deben considerar en esta formación de profesionales de la Educación Física, las asignaturas relacionadas al paradigma establecido para el modelo pedagógico de la carrera, que son las asignaturas que entregan la comprensión y la aplicación de los fundamentos de la $\mathrm{CMH}$ y cómo a partir de ésta preparación, poder actuar en el desarrollo de las funciones educativas en todos los niveles de enseñanza, entramados por las bases de la educación, para los proyectos pedagógicos de las escuelas, con un sin número de conocimientos, utilizados de manera práctica, de acuerdo con las necesidades y las exigencias, y en el momento real, reconociendo la Motricidad Humana como área científica, que se preocupa del estudio del ser humano y de sus acciones hacia la humanización. 
PORTES J.M.; ALMONACID F.A.A. \& CASTRO J.C.A. (2014). Reflexiones respecto de un plan de estudio para pedagogía en educación física con fundamentación paradigmatica en la ciencia de la motricidad humana. Mot. Hum. 15(2): 124-133.

Para el Área de formación General, están las asignaturas relacionadas a la formación personal del futuro docente, con una amplia mirada al desarrollo de la persona, con una fuerte base crítica, consciente y competente, con una visión particular de la historia y de las asignaturas sociales, teniendo contextos que permitan la adquisición de conocimientos, de hábitos de estudio y de investigación de manera sistemática y asî mantenerse actualizado en cuanto a los conocimientos técnicos, científicos y culturales, para prestar mejores servicios y contribuir al desarrollo de la profesión.

\section{La Educación Física en el marco de las nuevas bases curriculares chilenas}

A pesar que la Educación Física mundial se encuentra regida bajo los parámetros de la Federación Internacional de la Educación Física, y que el ideal de ella está ordenado en el Manifiesto Mundial de la Educación Física, la realidad de ésta área no corresponde a una intención general y única. Los contextos de la historia humana, los contextos sociales y culturales, específicos de cada país donde la Educación Física está inserta, evidencian las concepciones que han de moldear la Educación Física, y la Educación Física escolar. (Portela Guarín, 2001; Gambarotta y Emiliozzi, 2011).

Bajo esta perspectiva, la cultura social chilena se encuentra de manera directa, influenciada por las estructuras y bases curriculares de la Educación Física escolar en el país, de acuerdo a lo que establecen las bases curriculares de la disciplina. Lo anterior, en el bien entendido que estén acordes con los referentes internacionales de la entidad máxima del área (Portes Jr. Y Vargas, 2012). Las políticas públicas vinculadas al cuidado de la salud y a la prevención de enfermedades, tienen como foco de atención la obesidad y a los hábitos sedentarios de la población, componentes que están siendo considerados para establecer el currículo de la Educación Física escolar. (Cornejo Arméstica, et, al.,2011). Esta situación tiene relación directa con los fenómenos de aptitud física, niveles de acondicionamiento físico, ejercicio físico. (Costa, et al., 1996).
Esta propuesta curricular mantiene su relación con el paradigma biologicista (Castelani Filho, 2000), desenvolvimentista (Tani, et al., 1998) de la Educación Física escolar, higienista (cuerpo saludable) y eugenista (disciplinadora), que lleva dentro de su teoría, el ejercicio físico para la mantención y cuidados de la salud, con los objetivos pretendidos a través de resultados de test de acondicionamiento físico y de aptitud física, características del tiempo del militarismo. (Pacheco, 1998). La investigación realizada por Moreno y Álvarez (2010), confirma esta realidad, y concluye planteando que la creencia del profesorado de Educación Física, está centrada en un cuerpo máquina; salud, limitada al sujeto; Educación Física tecnocrática. Además, interpreta que el accionar del profesor, en lo relacionado a la capacitación, se aleja de manera considerable del campo de la educación y formación humana.

Lo opuesto a esta realidad, es que la Educación Física a nivel mundial, viene desarrollando propuestas más humanizadoras del proceso de formación en cuanto a salud y actividad física. Teniendo como referencia lo planteado por Manuel Sergio y a partir de sus investigaciones y publicaciones, muchos académicos, en diversos países, vienen adoptando las propuestas presentadas por la $\mathrm{CMH}$, justamente para buscar humanizar el accionar de la Educación Física escolar y hacerla más accesible a todos los estudiantes, porque considera el ser humano, con sus características personales, en todas las dimensiones que lo forman. Una Educación Física que no está restringida por números y valores de resultados de test $\mathrm{y}$ mediciones, pero sí, que sea una Educación Física que pueda humanizar las actividades deportivas y la actividad física; contribuir al desarrollo de la consciencia constructiva, construir significados en un mundo propio y compartido, es posible para todos, ser coherente y pertinente al individuo que la realiza; garantizar posibilidades de autorrealizarse, supliendo necesidades individuales. Todo esto, refiriéndose a lo que Sergio plantea, el cuerpo, el sujeto, no es un simple organismo, que puede ser medido, entrenado y evaluado; sino que es una red de intencionalidades y de infinitas posibilidades de comunicación con el mundo. (Sergio, 1995; Sergio, 1999; Sergio, 2000; Sergio, 2003 B). 
PORTES J.M.; ALMONACID F.A.A. \& CASTRO J.C.A. (2014). Reflexiones respecto de un plan de estudio para pedagogía en educación física con fundamentación paradigmatica en la ciencia de la motricidad humana. Mot. Hum. 15(2): 124-133.

La Universidad Autónoma de Chile en el plan de estudios de la carrera de Pedagogía en Educación Física, establecido bajo el paradigma de la $\mathrm{CMH}$; declara explícitamente que el futuro docente ha de ser responsable de transformar vidas a través del trabajo educativo y formativo de la Educación Física escolar. Cuenta con un perfil pedagógico capaz de trabajar con los medios y contenidos de la Educación Física, a partir de una mirada transformadora y renovada del fenómeno educativodeportivo, contribuyendo al proceso de humanización del ser humano, que transita por las aulas de las diferentes instituciones escolares. (Universidad Autónoma de Chile, 2012).

Desde este punto de vista, los procesos académicos realizados en la Universidad Autónoma de Chile por la unidad, se tensionan con las discusiones relacionadas a la incongruencia entre esta perspectiva humanizadora y la perspectiva higienista y eugenista de las mediciones SIMCE (Sistema de Medición de la Calidad de la Educación) de Educación Física y de las propuestas que están siendo establecidas, como políticas públicas de la actividad física y vida saludable. Esto no significa que la comprensión del fenómeno Educación Física, desarrollado en la Universidad, esté alejado de la comprensión de la realidad social y cultural chilena en relación al tema de la salud de la población. Pero si está acorde con muchos otros académicos, investigadores e instituciones, que han expresado razones en este sentido. (Cañoles Cañoles, 2011; Medina González, 2012; Cresp Barría, 2012).

La crítica que se hace no es a la realidad y a las decisiones tomadas en relación a los cambios en el curriculum escolar para la Educación Física; pero si, al rompimiento, una vez más, de hacer una Educación Física escolar que esté direccionada al desarrollo del estudiante de manera integral, buscando su humanización y trascendencia a partir de las acciones propuestas en el programa de estudio. Se observa que cada vez está más direccionada a la práctica de la actividad física y ejercicio físico, con la finalidad de desarrollar patrones que puedan ser medidos y calificados. No parece ser un proceso que busca la integralidad del proceso educacional y formativo, que considera el estudiante como un todo y las especificidades de cada uno de ellos, porque cuando se califica a partir de mediciones, se busca establecer resultados paramétricos que no son los más indicados para trabajar con la diversidad, la integración, la inclusión, existente en cada lugar educativo, y fundamentalmente al estímulo personal que produzca cambios positivos para una educación para la salud. Las tablas de clasificación en los test y mediciones, consideran patrones especiales, que en la mayoría de los casos, no se ajusta a los objetivos y a la realidad a alcanzar en la Educación Física escolar.

\section{A modo de conclusión}

Se reconoce la importancia de la Educación Física escolar como una herramienta de educación para la salud (FIEP, 2013). Se reconoce también, que la escuela desempeña un papel importante en las políticas públicas en este sentido. Pero no se comprende que se deba llevar a la Educación Física escolar a tener como su fundamento, la formación del estudiante en la realización de actividad física y ejercicio físico, en la "autonomía e independencia..., y en la búsqueda de la mejora de la condición física como factor de "calidad de la Educación Física" y como esperanza que a partir de ésta posible mejora, el estudiante adquiera un comportamiento hacia el cuidado de la salud a partir del ejercicio y actividad física.

De la misma manera, no se puede olvidar que a la vez que se establezcan las políticas gubernamentales de la Educación Física escolar para el cuidado de la salud, hay innumerables influencias que socavan cualquier trabajo educacional y formativo en esta área, que están liderados e incentivados por los medios de comunicación, como las drogas lícitas, el alcohol y el cigarrillo, las comidas rápidas y la alimentación basada en azúcares y grasas. También, la era tecnológica y todos los aparatos que son comercializados e incentivados para consumo y para "modernizar y tornar más fácil" la vida de las personas. Estos elementos juegan muy fuertemente en contra de los intentos de profesionales preocupados de la Educación Física en las escuelas y colegios. 
PORTES J.M.; ALMONACID F.A.A. \& CASTRO J.C.A. (2014). Reflexiones respecto de un plan de estudio para pedagogía en educación física con fundamentación paradigmatica en la ciencia de la motricidad humana. Mot. Hum. 15(2): 124-133.

Se puede pensar que la humanización en las políticas de la Educación Física escolar, es responsabilidad de ella y se "pone sobre los hombros" de ésta, la solución para los problemas sociales relacionados al sedentarismo y mala alimentación; debería ser dividida y responsabilizada con otros estamentos sociales. Se ha puesto en la Educación Física escolar la culpa de los malos resultados del SIMCE y de los índices de obesidad y sedentarismo social.

Clases bien desarrolladas, estructuradas y planificadas en deportes, juegos, actividades recreativas, mezclando ejercicios físicos y actividades físicas, en intensidades y volúmenes adecuados, pueden favorecer de manera positiva en la condición física del estudiante, sin perder los aspectos lúdicos, afectivos y sociales necesarios para que la Educación Física se mantenga en el proceso de humanización. Sin olvidar que una caminata diaria de una hora, no ha de ser la única estrategia para producir cambios fisiológicos en el individuo, que sean capaces de alterar su condición física. Hay otros factores que deben ser incorporados al cotidiano que permitan mejorar la condición física y la salud de la persona. No basta con hacer ejercicios en la clase de Educación Física como la única acción que será responsable por producir los cambios que las políticas públicas preconizan en beneficio de la salud.

Se terminan estas reflexiones, con algunas preguntas para extender a nuevas posibilidades de análisis y reflexión: ¿La sociedad necesita profesionales que comprendan el ser humano en su integralidad, o instructores de ejercicios que tienen vistas a los estándares establecidos en las tablas de los testes estadísticas? ¿La Educación Física escolar será más atrayente a los estudiantes, si fuere trabajada con más compatibilidad a la integralidad de ellos, o debe adaptarse a las políticas públicas basadas en estadísticas sociales? ¿Las carreras de formación en Educación Física deben seguir en el paradigma humanizador de la $\mathrm{CMH}$ o volver al mecanicismo de los modelos higienistas?

\section{REFERENCIAS BIBLIOGRÁFICAS}

Almonacid, A. (2012). "La educación física como espacio de transformación social y educativa: perspectivas desde los imaginarios sociales y la ciencia de la motricidad humana". Estudios Pedagógicos, 38:177-190.

Ardoy DN, Fernández-Rodríguez JM, Chillón Palma AEG, España-Romero V, JiménezPavón D, et al. (2010). Educando para mejorar el estado de forma física, estudio EDUFIT: antecedentes, diseño, metodología y análisis del abandono/adhesión al estudio. Rev. Esp. Salud Publica [Internet]. Apr [citado 16 Jun 2013]; 84(2):151-168. Disponible en: http://www.scielosp.org/scielo.php?script=sci _arttext\&pid=S113557272010000200004\&lng=en.

Ausubel D, Novak J, Hanesian H. (1983). Psicología Educativa: Un punto de vista cognoscitivo. $2^{\circ}$ Ed.,TRILLAS, México.

Betti M, Ferraz OL, Dantas LEPBT. (2011). Educação física escolar: estado da arte e direções futuras. Rev. bras. educ. fís. Esporte. dec; 25(esp.).

Boscatto JD, Kunz E. (2002). Didática Comunicativa: contribuições para a legitimação pedagógica da educação física escolar, R. da Educação Física. 2 trim.; 20(2):183-195.

Bougère G. A (1998). Criança e a Cultura Lúdica, Rev. Fac. Educ. jul/dic; 24(2).

Bracht VA. (1999).Constituição das Teorías Pedagógicas da Educação Física. Cadernos CEDES. ago; ano XIX(48).

Braid LMC, Machado MFAS, Aranha AC. (2012). Estado da arte das pesquisas sobre currículo em cursos de formação de de profissionais da área da saúde: um levantamento a partir de artigos publicados entre 2005 e 2011. Interface. jul/sep; 16(42).

Bruner JS. (1987). La Importancia de la Educación. Editorial Paidós, Barcelona.

Cañoles Cañoles FI. Sistema de Medición de la Calidad de la Educación Física en Chile: análisis crítico sobre el estado de la cuestión. EF Deportes Revista Digital [Internet]. 2011 Mar [Citado 16 Jun 2013]; año 16(154): [aprox. 1 p.]. Disponible en: http://www.efdeportes.com/efd154/sistemade-medicion-de-la-calidad-de-la-educacionfisica-en-chile.htm. 
PORTES J.M.; ALMONACID F.A.A. \& CASTRO J.C.A. (2014). Reflexiones respecto de un plan de estudio para pedagogía en educación física con fundamentación paradigmatica en la ciencia de la motricidad humana. Mot. Hum. 15(2): 124-133.

Carrizo ME, Rodríguez C. La Formación Inicial en Educación Física en España y Argentina. Revista Wanceulen E.F. Digital [Internet]. 2012 feb [citado 16 Jun 2013]; (9):[84-100]. Disponible en:http://www.wanceulen.com/efdigital.

Castelani Filho L. (2000). A educação física no Brasil: a história que não se conta. 5a, Papirus, Campinas.

Ministério de Educación Chile. Programa de Estudio, Quinto Año Básico, Educación Física, Gobierno de Chile; 2002.

Ministerio de Educación Chile. Bases Curriculares Educación Media, Educación Física. Gobierno de Chile; 2013.

Cordova A, Villa G, Sureda A, Rodriguez-Marroyo JA, Sánchez-Collado MP. (2012). Actividad física y factores de riesgo cardiovascular de niños españoles de 11-13 años. Rev Esp Cardiol. jul; 65(7):620-626.

Cornejo Arméstica M, Matus Castillo C, Vargas Contreras C. La Educación Física en Chile: una aproximación histórica. EF Deportes Revista Digital. [Internet]. 2011 oct [Citado 16 Jun 2013]; Año 16(161):[Aprox. 1 p.]. Disponible en: http://www.efdeportes.com/efd161/laeducacion-fisica-en-chile.htm.

Costa FC, Carvalho LM, Onofre MS, Diniz JÁ, Pestana C. (1996). Formação de Professores em Educação Física; concepções, investigação, prática. Lisboa, Portugal, Edição Faculdade de Motricidade Humana, Capítulo 3.

Cresp Barría MA, Ojeda Nahuelcura R, Machuca Barría C, Sánchez Villalobos E, Urrra S. Visión desde el área de Educación Física acerca de los elementos constitutivos del SIMCE como instrumento de medición de la calidad y su pertinencia con el curriculum. EF Deportes Revista Digital [Internet]. 2012 Mai [Citado 16 de Jun 2013]; Año 17(168):[aprox. 1 p.]. Disponible en: http://www.efdeportes.com/efd168/visiondesde-educacion-fisica-del-simce.htm.

Cuadra-Martínez D, Georgudis-Mendoza CN, Alfaro-Rivera RA. (2012). Representación social de deporte y educación física en estudiantes con obesidad. Rev. latinoam. cienc. soc. niñez juv. sep; 10(2):983-1001.

Dirección de Salud Integral de la Niñez y Adolescencia. (2012). Directrices de evaluación de niñas, niños y adolescentes para la actividad física pedagógica recreativa y deportiva escolar en Paraguay. Pediatr. abr; 39(1):47-56.

Erickson E. (1983). Infancia y Sociedad. HormePaidós, Buenos Aires.

Escalante Y, Backx K, Saavedra JM, GarcíaHermoso A, Domínguez AM. Relación entre actividad física diaria, actividad física en el patio escolar, edad y sexo en escolares de educación primaria. Rev. Esp. Salud Publica. [internet]. 2011 [citado 16 Jun 2013]; 85(5):[481-489]. Disponible en: http://www.msc.es/biblioPublic/publicaciones /recursos_propios/resp/revista_cdrom/vol85/v ol85_5/RS855C_481.pdf.

Farias E. (2008). Educação Física como Veículo de Acesso à Saúde, In: Vargas, A.; Pimentel, F.; Lames, C. (Orgs.) Questões do Esporte. Bruner, Rio de Janeiro.

Federación Internacional de Educación Física (FIEP). Manifiesto Mundial de educación Física [Internet]. 2013 Jun [Citado 16 Jun 2013]. Disponible en: www.fiep.org.

Figueredo González JM, González Medel L, Ortiz Tamayo J. (2003). El Juego como vía que tienen los niños de conocer el mundo que están obligados a transformar. Revista Electrónica Granma Ciencia. sep/dic; 7(3).

Freire ES, Silva SAPS, Miranda MLJ. (2011). Valores como Conteúdo da Educação Física Escolar: perspectiva a partir da Motricidade Humana. Revista Brasileira de Ciência e Movimento. 19(4):89-96.

GalakI EL, Gambarotta EM. (2011). Conquista, confirmación y construcción del cuerpo: una propuesta para el estudio de las prácticas corporales a partir de la epistemología de Pierre Bourdieu. Rev. Bras. Ciênc. Esporte. oct/dec; 33(4).

Gambarotta EM, Emiliozzi MV. (2011). Problemas de Educación Corporal: coordinadas de un mapa presente y desafíos a futuro. Educación Física y Ciencia. año 13:181-186. 
PORTES J.M.; ALMONACID F.A.A. \& CASTRO J.C.A. (2014). Reflexiones respecto de un plan de estudio para pedagogía en educación física con fundamentación paradigmatica en la ciencia de la motricidad humana. Mot. Hum. 15(2): 124-133.

González J, Garcés de los Fayos EJ, García Dantas A. (2012). Indicadores de bienestar psicológico percibido en alumnos de Guedes C.M. (2000). A Educação Física e os Mistérios do seu Tempo. Revista Brasileira de Ciências do Esporte, jan/mai; 21(2/3).

Kolyniak Filho C. (2008). Educação Física: uma (nova) introdução. $2^{a}$ ed., EDUC, São Paulo.

Lagos Hernández RI. (2011). La Motrcidad Nuestra de cada Día: análisis de los alcances conceptuales de la Motricidad Humana como ciencia emergente. Rev. Motricidad humana. jul; 12(1):51-57.

Lima HLA. (2000). Pensamento Epistemológico da Educação Física Brasileira: das controvérsias acerca do estatuto científico. Rev. Bras. De Cienc. do Esp. 21(2):95-102.

Machado TCJ, Vargas A. (2011). Historia de la Humanidad y el Deporte: un análisis desde una perspectiva politico-social. Rev. Motricidad Humana. ago-dic; 12(2).

Marcelino NC. (2001). Lúdico, Educação e Educaão Física. UNIJUí, Ijuí.

Marcon D, Graça ABS, Nascimento JV. (2012). Práticas Pedagógicas como Cenário para a Construção do Conhecimento Pedagógico do Conteúdo dos Futuros Professores de Educação Física. Rev. Educ. Fis. 2 Trim.; 23(2):295-306.

Maslow AH. (1943). A Theory of Human Motivation, Psychological Review. 50:370396.

Medina González CT. SIMCE de Educación Física: primeros resultados y primeros interrogantes. EF Deportes Revista Digital [Internet]. 2012 Mar [Citado 16 de Jun 2013]; Año 15(166):[aprox. 1 p.]. Dis`ponible en: http://www.efdeportes.com/efd166/simce-deeducacion-fisica-2010-primerosresultados.htm.

Moreno A, Álvarez N. (2010). Creencias del profesorado universitario de educación física en relación a los conceptos de cuerpo y salud. Estudios Pedagógicos XXXVI. (1):159-175.

Moreira WW. Carbinatto MV (2006). Bases Epistemológicas, a Educação Física e o Esporte: posibilidades. Rev. Bras. Educ. Fís. Esp. 2006 set, Supl 20(5):129-30.

Neira MG. (2012). Alternativas existem! Análise da Produção Científica em Dois Períodos
Educación Física, Rev. psicol. deport. 21(1):183-187.

Brasileiros sobre a Docência em Educação Física, Movimento. jan/mar; 18(1):241-257.

Oliveira GM, Porpino KO. (2010). Ginástica Rítmica e Educação Física Escolar: perspectivas críticas em discussão. Pensar a Prática. mai/ago; 13(2):1-18.

Pacheco AJP. (1998). Educação Física Feminina: uma abordagem de gênero sobre as décadas de 1930 e 1940. Revista da Educação Física. 9(1):45-52.

Pérez Gallardo JS. (2004). Educação Física: contribuição à formação profissional. $4^{\mathrm{a}} \mathrm{ed}$, UNIJUÍ, Ijuí.

Piaget J. (1969). Psicología y Pedagogía, Ariel, Barcelona.

Portela Guarín H. (2001). Paradigmas del Cuerpo en la Educación Física. EF Deportes Revista Digital [Internet]. 2001 Nov [Citado 16 Jun 2013]; año 7(42):[aprox. 1 p.]. Disponible en: http://www.efdeportes.com/efd42/paradig.htm

Portes Jr. M, Vargas ALS. (2012). Relación entre el Curriculum en Carreras de Formación de Profesionales de Educación Física y el Manifiesto Mundial de la Educación Física. Revista Motricidad Humana. julio-diciembre; 13(2):68-73.

Rigo LC, Ribeiro GM, Hallal PC. (2011). Universidade na Diversidade: desafios para a Educação Física no século XXI. Revista Brasileira de Atividade Física e Saúde. 16(4).

Rivero IV. (2012). El Juego desde la Perspectiva de los Jugadores: una investigación para la didáctica del jugar en Educación Física. Revista Educación física y deporte. 31(1):925928.

Rogelin S, Saraiva MC, Bergero VA. (2010). Brasil e Argentina: estudo comparativo sobre conteúdos da educação física escolar e questões de gênero, In: Matielo Júnior, E, Capela, P, Breilh, J. Ensaios alternativos latino-americanos de educação física, esportes e saúde. Copiart, Florianópolis.

Rogers C. (1961). El Proceso de Convertirse en Persona: Mi técnica terapéutica. Paidós, Buenos Aires. 
Sacardo MS, Hayashi MCPI. (2010). A Pesquisa em Educação Física na Interface com a Educação na Região Centro-Oeste do Brasil: uma análise epistemológica e bibliométrica. Anais do V Colóquio de Epistemologia da Educação Física, outubro 22-23, Maceió-AL, Brasil.

Savarí JR. (2012). La Praxiología Motriz: presente, pasado y futuro. Entrevista a Pierre Parlebas. Movimento. jan/mar; 18(1):11-35.

Sérgio M. (1995). Motricidad Humana: contribuições para um paradigma emergente. Instituto Piaget, Lisboa; P. 156.

Sergio M. (1996). Epistemología da Motricidade Humana. Lisboa: Edições FMH.

Sérgio M. (1999). Um Corte Epistemológico: da Educação Física à Motricidade Humana. Instituto Piaget, Lisboa. P. 284.

Sérgio M. (2000). Para uma Epistemologia da Motricidade Humana, $3^{\mathrm{a}}$ Ed., Compendium, Lisboa. P. 176.

Sergio M. (2003). Para uma Nova Dimensão do Desporto. Instituto Piaget, Lisboa. A. 284.

Sérgio M. (2003). Algunas teses sobre o desporto. Compendium, Lisboa. B.

Silva AA. (2012). A importância do lúdico para o desenvolvimento da criança. 2012. [Monografia]. Universidade de Brasília, Monte Negro.

Souza Neto S, Alegre NA, Hunger D, Pereira JM. (2004). A Formação do Profissional de Educação Física no Brasil: uma história sob a perspectiva da legislação federal no século XX. Revista Brasileira de Ciências do Esporte. jan; 25(2):113-128.

Tani G, Manoel EJ, Kokubun E, Proença JE. (1998). Educação física escolar: fundamentos de uma abordagem desenvolvimentista. EPU: EDUSP, São Paulo.

\section{Enviar Correspondencia a:}

Moacyr Portes Junior.

Universidad Autónoma de Chile, sede Talca, Chile.

5 Poniente, 1670, Talca, Chile.

Email-mportesj@uautonoma.cl.
Trigo E, Montoya H (2006). Teorías que Fundamentan la Ciencia de la Motricidad Humana. IV Coloquio internacional de Pedagogía y Currículo. 17-20 octubre. Paipa:Colombia.

Trigo E, Montoya H. (2009). Motricidad Humana: política, teorías y vivencias. Léeme, Instituto Internacional del Saber, España-Colombia.

Ubirajara O. A Ciência da Motricidade Humana: Perspectiva epistemológica em Piaget. Instituto Piaget. [Internet]. 1999 [Citado 18 Feb 2013]; [ [280 p.] Disponible e: http://www.google.com.br/\#hl=ptBR\&gs_rn= 1\&gs_ri=serp\&pq=ubirjara\%20oro\%201994 $\& \mathrm{cp}=77 \& \mathrm{gs} \_\mathrm{id}=14 \& \mathrm{xhr}=\mathrm{t} \& \mathrm{q}=\mathrm{A} \% 20 \mathrm{Ci} \% \mathrm{C} 3$ $\%$ AAncia\%20da\%20Motricidade $\% 20$ Humana $\% 20$ Perspectiva\%20epistemol\%C3\%B3gica\% 20em $\% 20$ Piaget $\% 20$ download $\& p f=p \&$ tbo $=\mathrm{d}$ \&sclient $=$ psyab\&oq $=\mathrm{A}+\mathrm{Ci} \% \mathrm{C} 3 \%$ AAncia $+\mathrm{da}$ + Motricidade+Humana+Perspectiva+epistem ol\%C3\%B3gica+em+Piaget+download\&gs_1 $=\& p b x=1 \& b a v=o n .2, o r . r \_g c . r \_p w . r \_q f . \& f p=$ 6e6a7a9468c6e $849 \&$ biw $=800 \&$ bih $=509$.

Universidad Autónoma de Chile. (2012). Informe de Autoevaluación, Carrera de Pedagogía en Educación Física.

Usabiaga Arruabarrena O, Matos I, García D. (2012). La tradición lúdica en el currículum educativo. Los juegos y deportes de pelota en Educación Física: una propuesta comprensiva. Apunts. Educación Física y Deportes 1er trim.; (107):35-44.

Veloso EL. (2010). Educação Física e Epistemología: a cientificidade como uma dimensão cultural, Rev. Bras. Cienc. Esporte. mai; 31(3):79-93.

Vygotsky L S. (1978). Pensamiento y lenguaje. Paidós, Madrid. 